\title{
Interaction between an elastic structure and free-surface flows: experimental versus numerical comparisons using the PFEM
}

\author{
S. R. Idelsohn · J. Marti · A. Souto-Iglesias · E. Oñate
}

Received: 20 November 2007 / Accepted: 16 January 2008 / Published online: 12 February 2008

(c) Springer-Verlag 2008

\begin{abstract}
The paper aims to introduce new fluid-structure interaction (FSI) tests to compare experimental results with numerical ones. The examples have been chosen for a particular case for which experimental results are not much reported. This is the case of FSI including free surface flows. The possibilities of the Particle Finite Element Method (PFEM) [1] for the simulation of free surface flows is also tested. The simulations are run using the same scale as the experiment in order to minimize errors due to scale effects. Different scenarios are simulated by changing the boundary conditions for reproducing flows with the desired characteristics. Details of the input data for all the examples studied are given. The aim is to identifying benchmark problems for FSI including free surface flows for future comparisons between different numerical approaches.
\end{abstract}

Keywords Fluid-Structure Interaction (FSI) .

Free surface flows · Fluid dynamics

S. R. Idelsohn $(\bowtie)$

International Centre for Numerical Methods in Engineering

(CIMNE), Institució Catalana de Recerca i Estudis

Avançats-ICREA, Barcelona, Spain

e-mail: sergio@cimne.upc.edu

S. R. Idelsohn · J. Marti

International Centre for Computational Methods in Engineering

(CIMEC), National University of Litoral (UNL),

Santa Fe, Argentina

A. Souto-Iglesias

Naval Architecture Department (ETSIN),

Technical University of Madrid (UPM), Madrid, Spain

E. Oñate

International Centre for Numerical Methods in Engineering

(CIMNE), Technical University of Catalonia (UPC),

Barcelona, Spain

\section{Introduction}

The availability of sufficient computer power, together with the maturity of the tools for CFD analysis, open the way to the simulation of flow problems of increasing complexity. Between the many potential applications, the simulation of Fluid-Structure Interactions (FSI) problems including freesurface flows represents a particularly interesting case. The challenge is in this case connected both to the inherent difficulty to solve FSI problems with the simulation of a highly unsteady flow with a rapid variation of the fluid domain.

There exist in the literature many comparisons between experiments and numerical solutions for FSI problem without free surfaces; there also exist some publications concerning the comparison between experimental and numerical solutions for fluid mechanics problems with free surface flows. Nevertheless, one of the most important cases in which the fluid flow including the free surface motion interact with elastic structures has not been well documented, and it is very difficult to find experimental results to compare and to check the accuracy of the different methods developed.

The objective of this work is to present a set of three examples for FSI problems including free surface flows solved experimentally and numerically in order to have different benchmarks for comparison with different numerical models.

From the numerical point of view, different methods have been devised over the last years to deal with this challenge. One of the methods developed in recent years to address the computational challenges involved in this class of problems is the Mixed Interface- Tracking/ Interface- Capturing Technique (MITICT) [2]. The MITICT was introduced for FSI with multiple fluids or free-surface flows. It was successfully used in a number of test problems [3,4]. In this work, only a comparison with the Particle Finite Element Method (PFEM) will be performed. A complete description of PFEM may be 
found in [5]. Only a light overview of this numerical method will be described in the next section for comprehensive.

A comprehensive comparison of the PFEM method with experimental results including mesh refinement and convergence test may be found in [6]. In this reference only fluid flow problems are compared without any elastic structure interaction. The extension to deal with the elastic deformation of a structure in a fluid is the objective of this work.

\section{Numerical simulation}

Different methods have been devised over the years to deal with transient free surface problems. A first category of algorithms is based on the idea of tracking the evolution of a free surface defined with the help of a smooth distance function (Level Set) [7], or of a scalar value representing the quantity of fluid in a given area. This is the basis of the Volume of Fluid (VOF) technique. This sealar function is convected according to the flow velocity field once a suitable discretization of the space is provided. This allows using existing Eulerian codes and justifies the success of the VOF method in the CFD
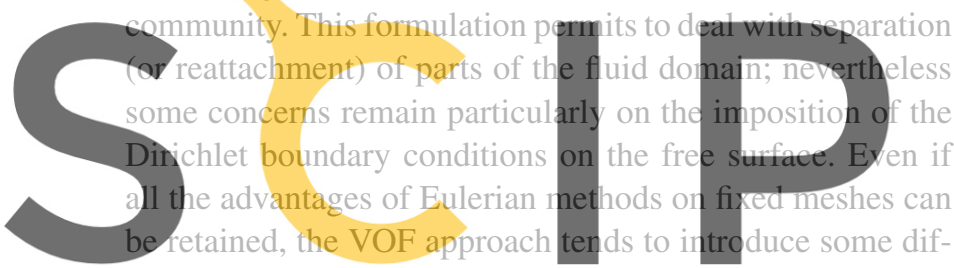

fusion in the position of sharp interfaces (see for examples Zalesak's circle benchmark [8]. While a. number enhanced

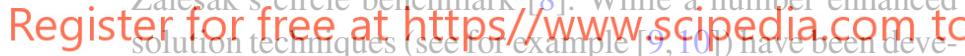
loped in recent years to increase the accuracy of the VOF-type methods, there is still need for development in this area.

An alternative formulation, known as Smooth Particle Hydrodynamics (SPH), allows a Lagrangian simulation of a number of particles through the use of a simple meshless technology [11]. This technique, which is raising an increasing interest in the scientific community due to its simplicity and computational efficiency, faces however some severe drawbacks. First it has troubles in representing constant functions (it is not a partition of unity) which implies problems in proving the convergence. Secondly its application is appealing as long as an explicit formulation for the fluid can be used, which makes it unattractive for truly incompressible flows [12].

The possibility exists to blend the advantages of "Particle" methods with finite element (FE) methods. The PFEM achieves this result by convecting in a Lagrangian way the fluid "particles" while redefining at the beginning of each step a new mesh. This allows to reproduce very accurately the convection of the nodes and to impose the Dirichlet conditions in a natural way. Further, all the convergence results can be inherited from the FEM which guarantees the reliability of the computational predictions [5].

The PFEM treats the mesh nodes in the fluid domain as particles which can freely move and even separate from the main fluid domain representing, for instance, the effect of water drops. A finite element mesh connects the nodes defining the discretized domain where the governing equations are solved in the standard FEM fashion. The PFEM is the natural evolution of recent work of the authors for the solution of FSI problems using Lagrangian finite element and meshless methods $[5,13]$.

An obvious advantage of the Lagrangian formulation is that the convective terms disappear from the fluid equations. The difficulty is however transferred to the problem of adequately (and efficiently) moving the mesh nodes. We use innovative mesh regeneration procedure blending elements of different shapes using an extended Delaunay tesselation $[14,15]$.

The need to properly treat the incompressibility condition in the fluid still remains in the Lagrangian formulation. The use of standard finite element interpolations may lead to a volumetric locking defect unless some precautions are taken $[16,17]$. In our work the stabilization via a finite calculus

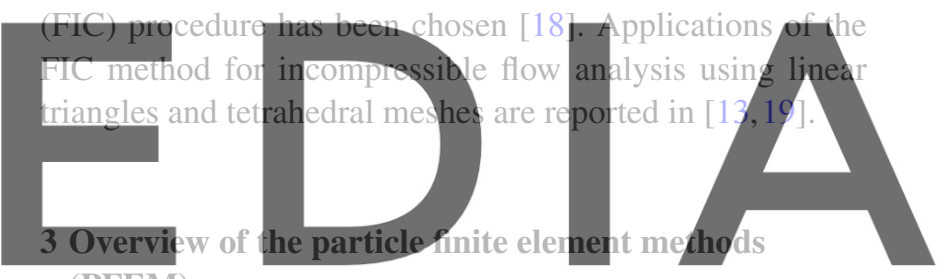

(PFEM)

\section{downloag the version without the watermark}

domains. The moving particles interact with the solid boundaries thereby inducing the deformation of the solid which in turn affects the flow motion making the problem fully coupled. In the PFEM, both the fluid and the solid domains are modeled using an updated Lagrangian formulation. That is, all variables in the fluid and solid domains are assumed to be known in the current configuration at time $t$. The new set of variables in both domains is sought for in the next or updated configuration at time $t+\Delta t$. The finite element method (FEM) is used to solve the continuum equations in both domains.

Hence a mesh discretizing these domains must be generated in order to solve the governing equations for both the fluid and solid problems in the standard FEM fashion.

We note that the nodes discretizing the fluid and solid domains are viewed as material particles whose motion is tracked during the transient solution. This is useful to model the separation of fluid particles from the main fluid domain and to follow their subsequent motion as individual particles with a known density, an initial acceleration and velocity and subject to gravity forces. It is important to note that each 
Fig. 1 Sequence of steps to update a "cloud" of nodes from time $n\left(t=t_{n}\right)$ to time

$n+1\left(t=t_{n+\Delta t}\right)$

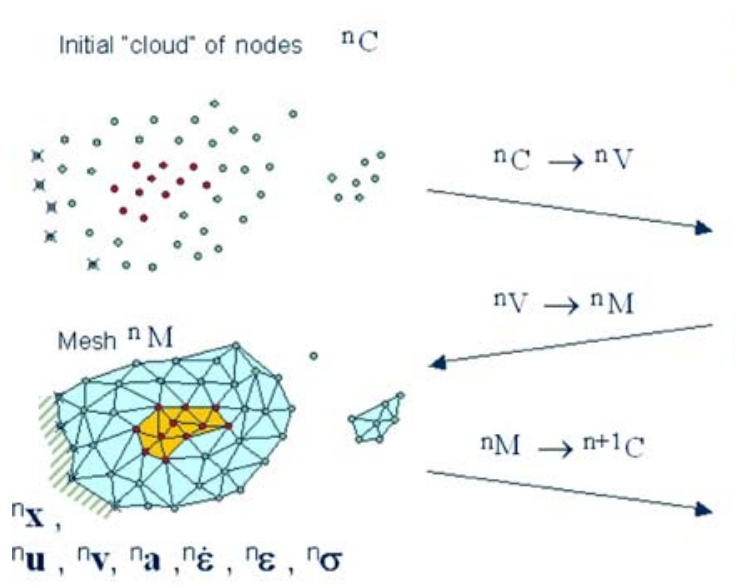

- Solid node

- Fluid node

*ixed boundary node
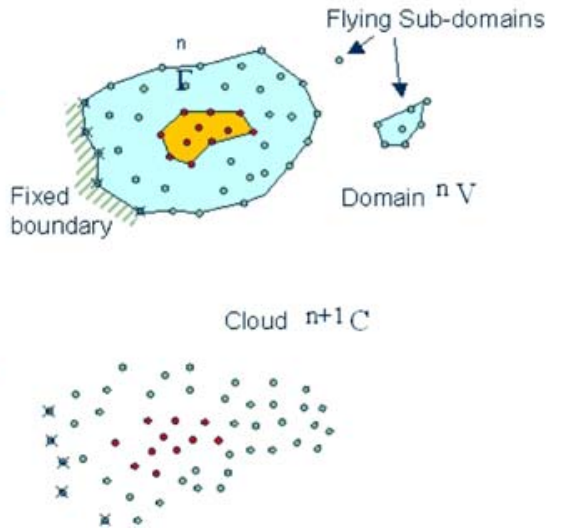

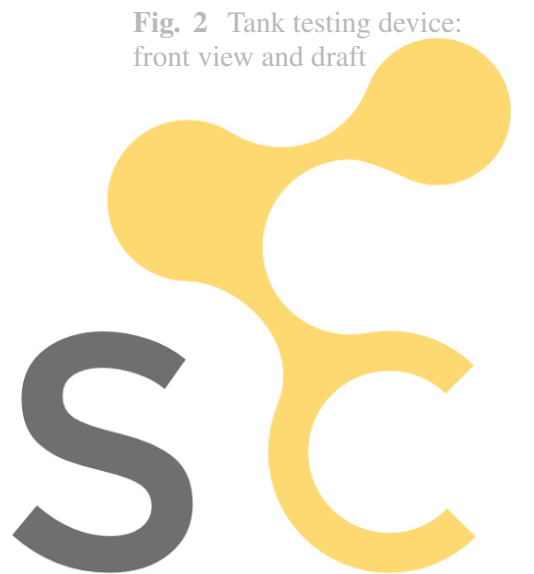

particle is a material point characterized by the density of Register folid free fluid domain to which it belongs. The mass of

different material points over the domain. The quality of the

numerical solution depends on the discretization chosen as in the standard FEM.

Adaptive mesh refinement techniques can be used to improve the solution in zones where large motions of the fluid or the structure occur. For clarity purposes we will define the collection or cloud of nodes $(C)$ pertaining to the fluid and solid domains, the volume $(V)$ defining the analysis domain for the fluid and the solid and the mesh $(M)$ discretizes both domains.

1. The starting point at each time step is the cloud of points in the fluid and solid domains. For instance $C$ denotes the cloud at time $t=t_{n}$ (Fig. 1).

2. Identify the boundaries for both the fluid and solid domains defining the analysis domain $V$ in the fluid and the solid. This is an essential step as some boundaries (such as the free surface in fluids) may be severely distorted during the solution process including separation and re-entering of nodes. The Alpha Shape method [20] is used for the boundary definition.

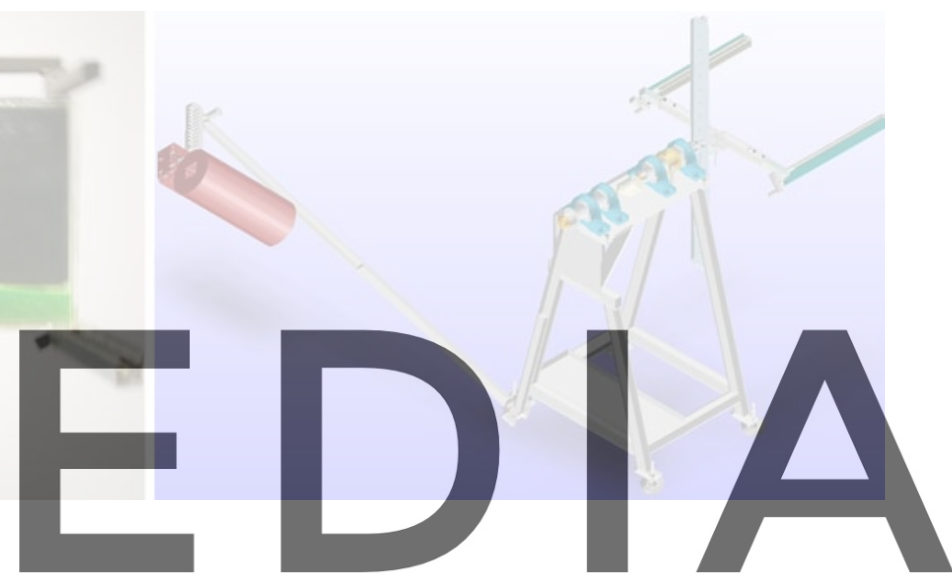

3. Discretize the fluid and solid domains with a finite downloment mesh $M$. In our work we use an innovative tessellation [15].

4. Solve the coupled Lagrangian equations of motion for the fluid and the solid domains. Compute the relevant state variables in both domains at the next (updated) configuration for $t+\Delta t$ : velocities, pressure and viscous stresses in the fluid and displacements, stresses and strains in the solid.

5. Move the mesh nodes to a new position $C^{n+1}$ where $n+1$ denotes the time $t_{n}+\Delta t$, in terms of the time increment size. This step is typically a consequence of the solution process of step 4

6. Go back to step 1 and repeat the solution process for the next time step.

\section{Experimental model}

The experimental data used for the comparison are taken from the laboratory tests carried out specifically for this study using the tank testing facilities at ETSIN-UPM. The experimental equipment is schematically represented in Fig. 2. 

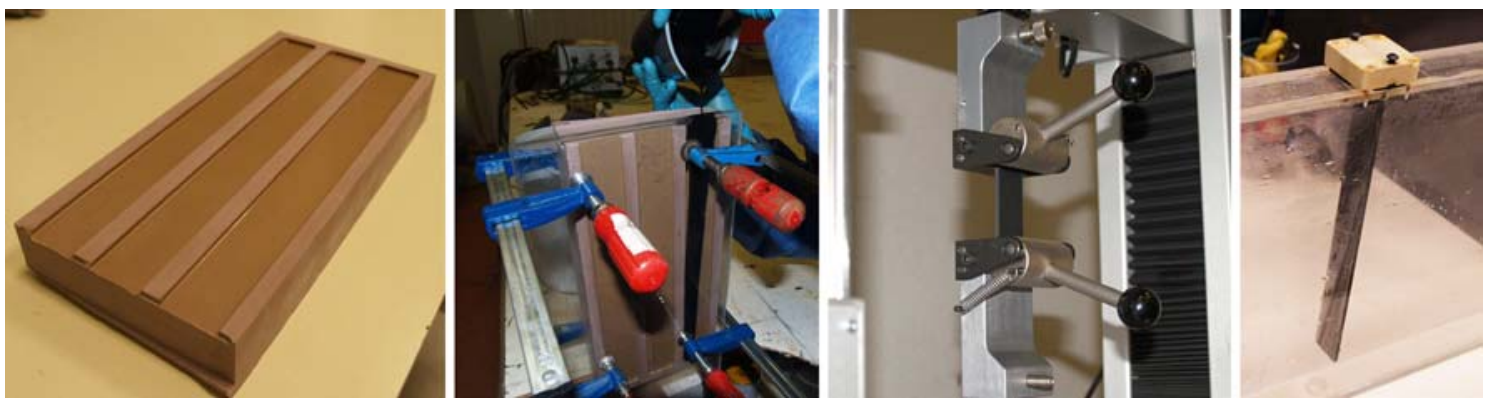

Fig. 3 Mould for the probes, syntethizing process, traction test and anchorage

It is composed of a structure that holds a tank and an electrical engine that produces a harmonic rolling motion on the moving part of the structure, which embraces the tank with the liquid inside. The system incorporates a high precision torquemeter with a $200 \mathrm{Nm}$ range because it is routinely used for the design of the passive anti-roll tanks for fishing vessels. It has been used previously aimed at providing validation data for CFD studies, both in terms of free surface shape as well as in terms of the effect of the liquid with respect to the tank motion, by measuring the torque produced by the motion of the liquid [21].

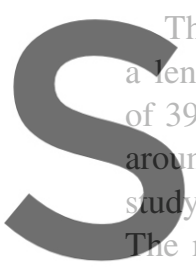

he tank, that is made of meth ength of $609.0 \mathrm{~mm}$, a height $39.0 \mathrm{~mm}$. The container can $\mathrm{m}$ the fixed point in order to the fixed point is the center magnitude of maximum ang
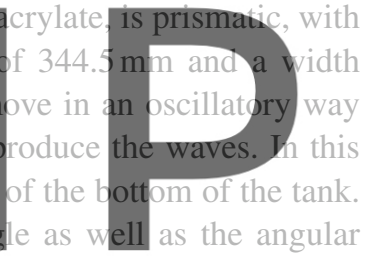
speed can be regulated, aimed at matching the critical slo-

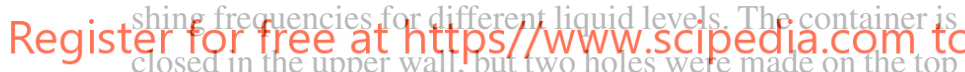
in order to allow the air to circulate freely without affecting the liquid behavior.

On the bottom wall or in the upper one, an elastic beam may be clamped to interact with the incompressible fluid. The beams used have a thickness of $4 \mathrm{~mm}$ and width of $33.2 \mathrm{~mm}$ which is enough to simulate a 2D flow without touching the lateral walls. The minimum admissible gap was found to be $2.9 \mathrm{~mm}$ for the longest configuration of the probes $(287.1 \mathrm{~mm})$. It would be desirable to have a smaller gap with the tank walls but due to the flexibility of the material, the rubber beam is prone to slightly bend on the front direction driven by capillarity and surface tension effects, thus touching the tank walls and invalidating the experiment. On top of this, it was discovered that smaller gaps made it extremely difficult the positioning at the anchorage.

The material for the probes clamped to the bottom is a dielectric polyurethane resin, whose trademark is AXSON RE 11820-(9). It was specifically syntethized for these tests by mixing the components and carefully filling a mould milled with the probe dimensions (Fig. 3). The density of the

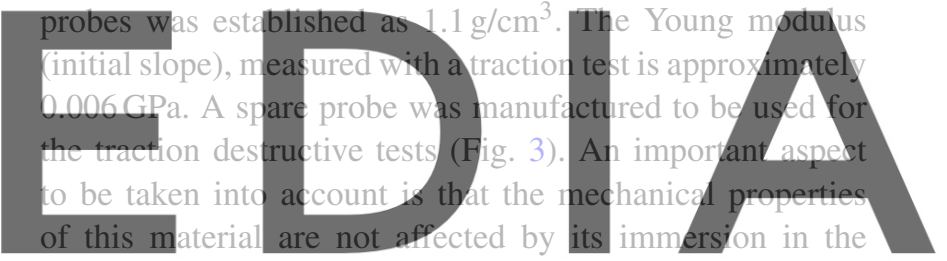

liquids during the sloshing tests. For the probes clamped to

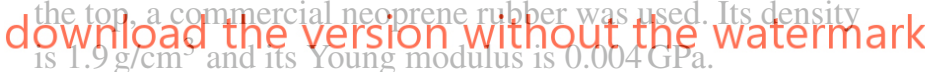

An anchorage piece was designed and milled to clamp the probe to the tank roof or bottom guaranteeing both a very good bending restriction at the base, as well as an accurate leveling of the piece at the bottom/top of the tank (see Fig. 3). In this way, the flow is not significantly affected on the vicinity of the probe by the anchorage. A hole was prepared in the tank to receive the anchorage piece.

Regarding the liquids, fresh water and a commercial sunflower oil were used. The temperature of the tests was $23^{\circ} \mathrm{C}$.
Fig. 4 Clamped elastic beam in shallow oil: initial geometry and angle of the container versus time
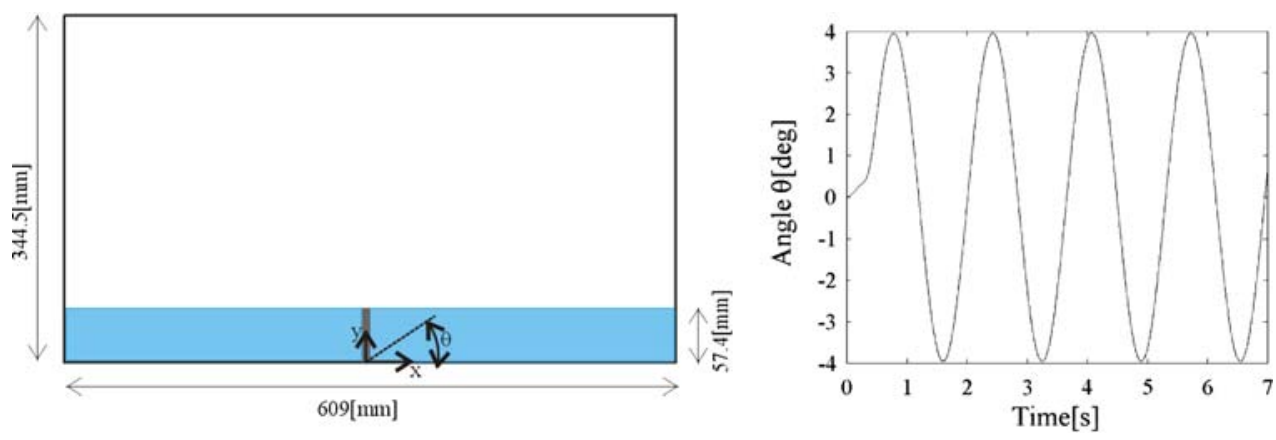

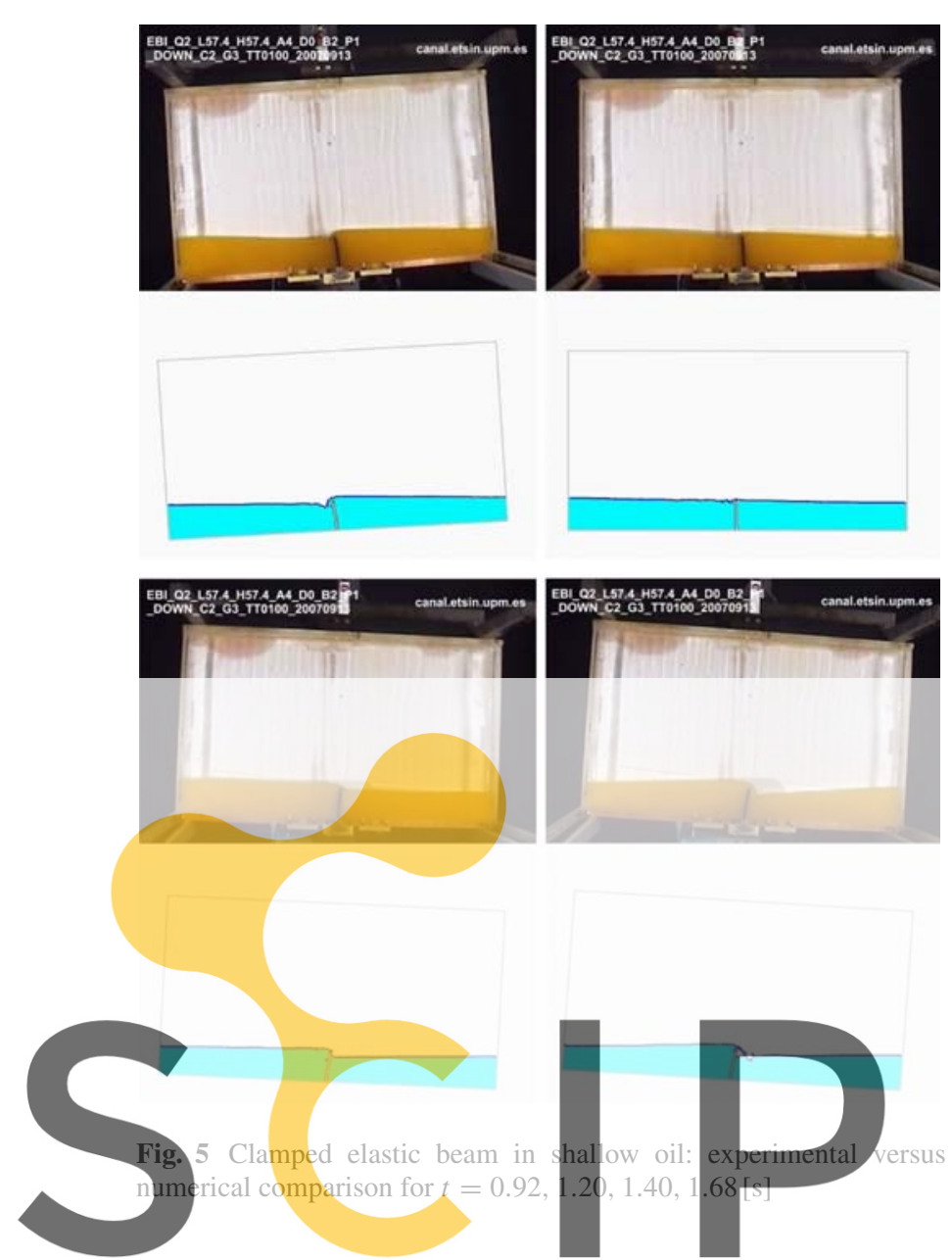

The sunflower oil density was 0.917. The kinematic visco-

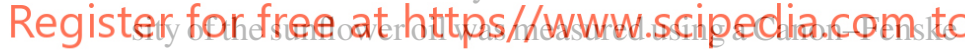
viscosimeter running a series of tests at 23,40 and $50^{\circ} \mathrm{C}$ and by extrapolating the tabulated constants for the viscosimeter that corresponded to the latter temperatures. At $23^{\circ} \mathrm{C}$ its value is $50 \mathrm{cst}\left(5 \mathrm{e}-5 \mathrm{~m}^{2} / \mathrm{s}\right)$. This means that the Reynolds number corresponding to the gap will be in principle 50 times smaller for the sunflower oil. The liquid levels considered were the same as the probe lengths. This means for all the probes there is one water level that corresponds exactly to the probe length, which is a limit case worth studying.

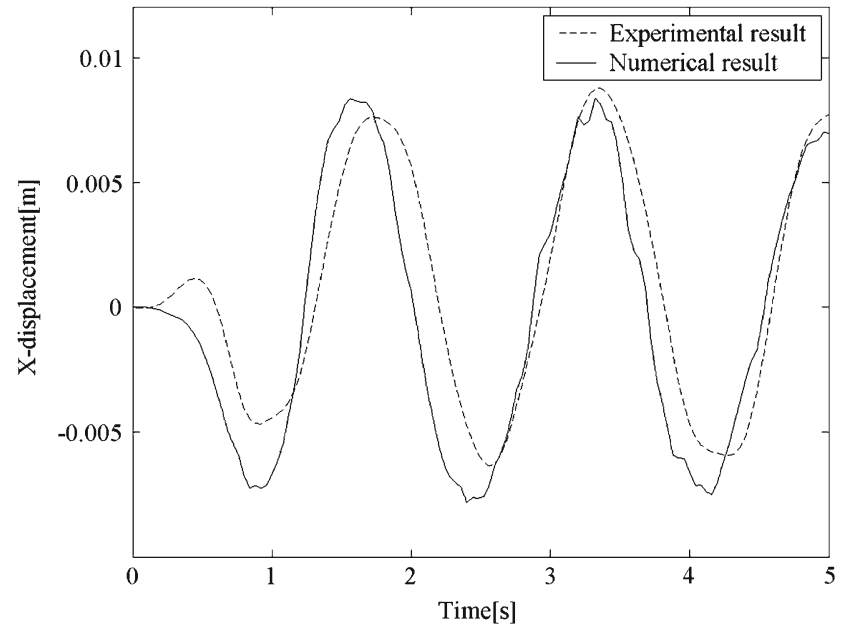

Fig. 6 Clamped elastic beam in shallow oil: comparison of the displacement in $X$ direction

A computer program was implemented aimed at measuring the total displacement of the elastic beam at different heights. The program facilitates the analysis of the individual frames obtained from a conventional video register of the experiment. For the short beam cases, only the displace-

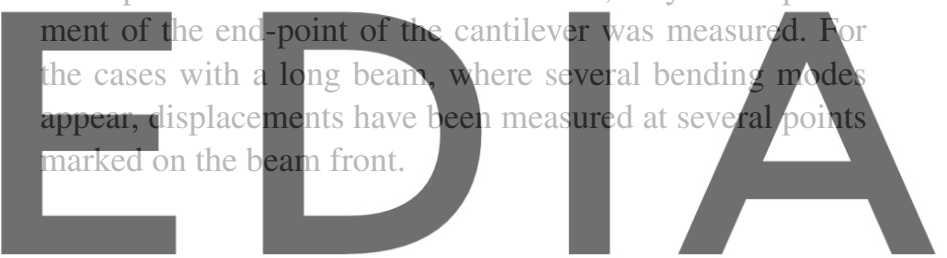

5 Experimental versus numerical comparisons

\section{download the version without the watermark}

5.1 Clamped elastic beam immersed in a shallow oil flow

The first two examples consist in a clamped beam of different lengths immersed in sunflower oil. Figure 4 represents the geometry and the angular displacement of the first example. The numerical model has 15,480 fluid particles and a total of 16,939 nodes including the solid and the fluid part. The average time step used was equal to $0.0025 \mathrm{~s}$. Snapshots of different instances of the experiment are shown and compared
Fig. 7 Clamped elastic beam immersed in deep oil flow: initial geometry and angle of the container versus time
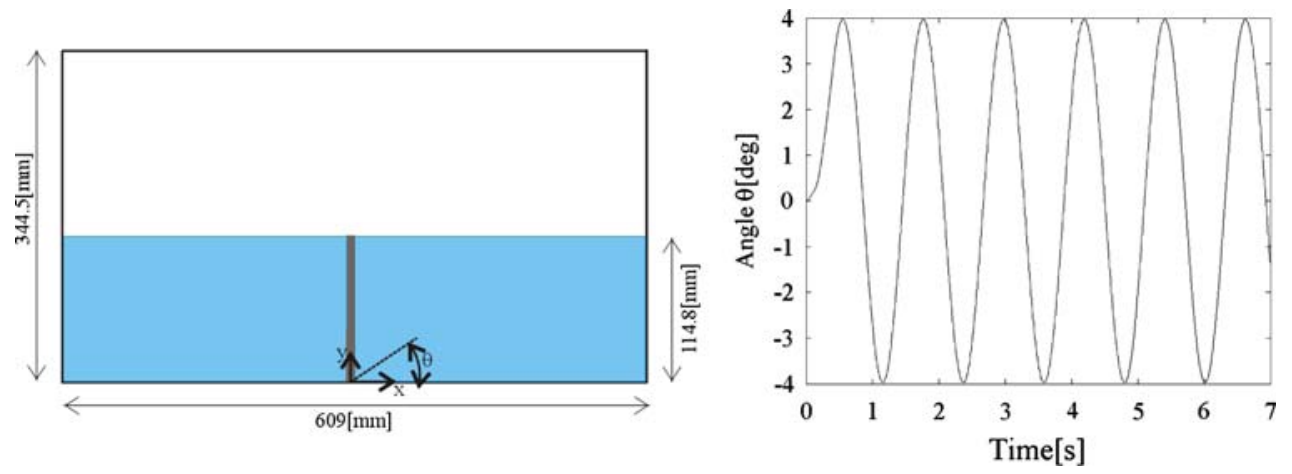

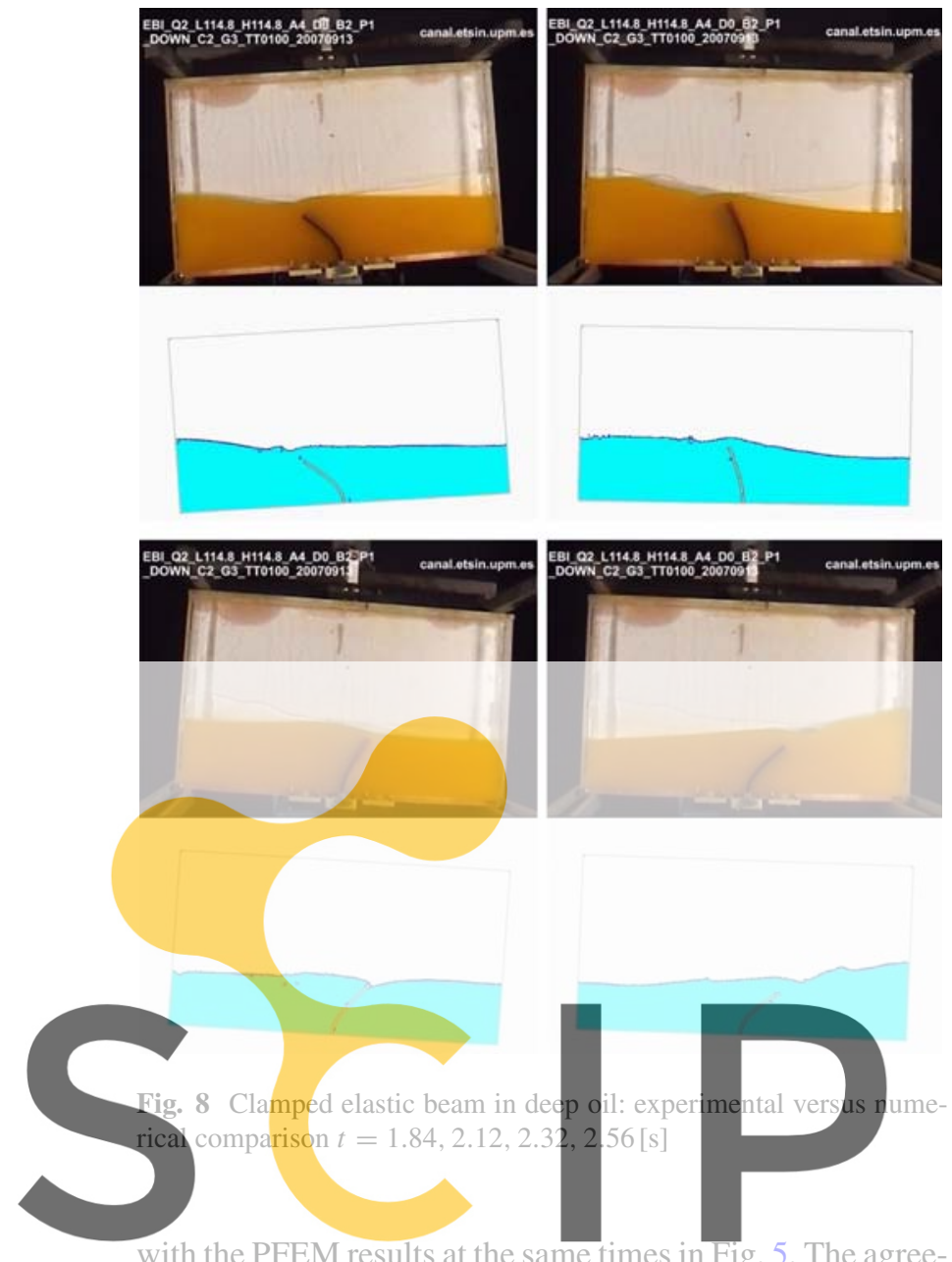

with the PFEM results at the same times in Fig. 5. The agree-

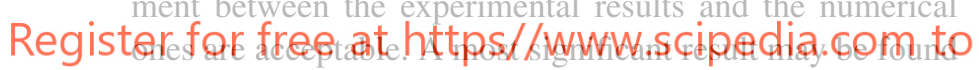
in Fig. 6 were the maximum $X$ displacement corresponding to the end point of the beam has been represented for both: the experimental and the numerical solution.

\subsection{Clamped elastic beam immersed in deep oil flow}

The second example is similar to the previous one but with a more deep oil flow. The geometry and the motions of the container are shown in Fig. 7. In this example, the mesh has a

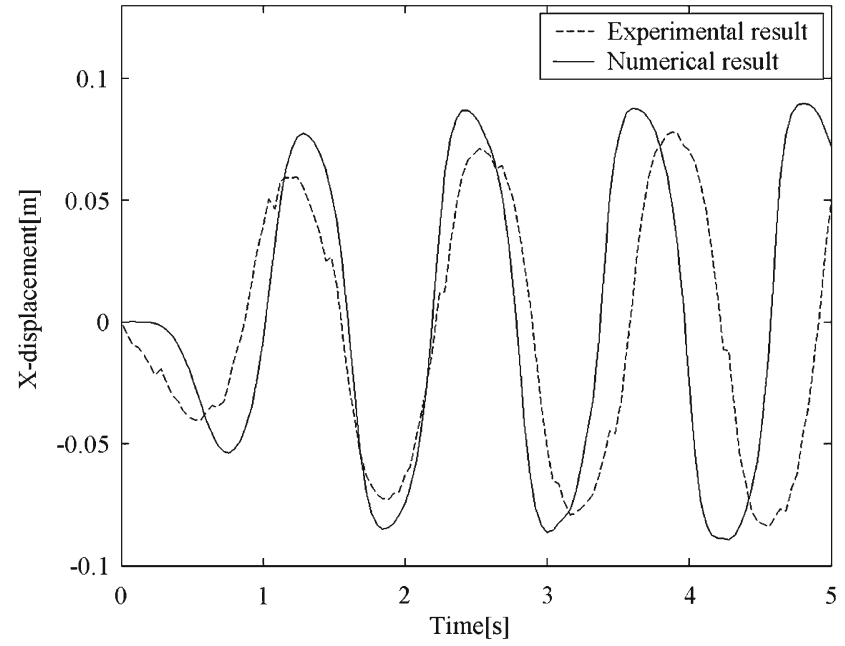

Fig. 9 Clamped elastic beam immersed in deep oil flow: comparison of the displacement in $X$ direction

total of 16,731 nodes with 15,371 nodes placed out the fluid part.

Figures 8 and 9 show the free surface and the beam displacement of the end point at different time steps respectively.

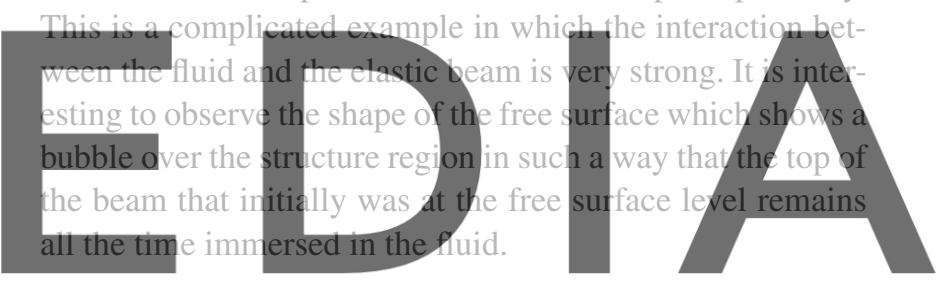

\section{downtoag the version without whe watermark}

This is the most difficult and impressive example. Now, the beam in hanging from the upper wall in such a way that the interaction with the fluid can be attained only due to the waves produced. Otherwise there is not interaction.

Figure 10 show the initial geometry and the motions of the container. The amount of particles used for the numerical solution was 16,924 and time step was equal to $0.0025 \mathrm{~s}$. Snap shots of the different instances of the experiment versus the PFEM results are shown in Fig. 11.
Fig. 10 Hanging elastic beam with shallow water: initial geometry and angle of the container versus time
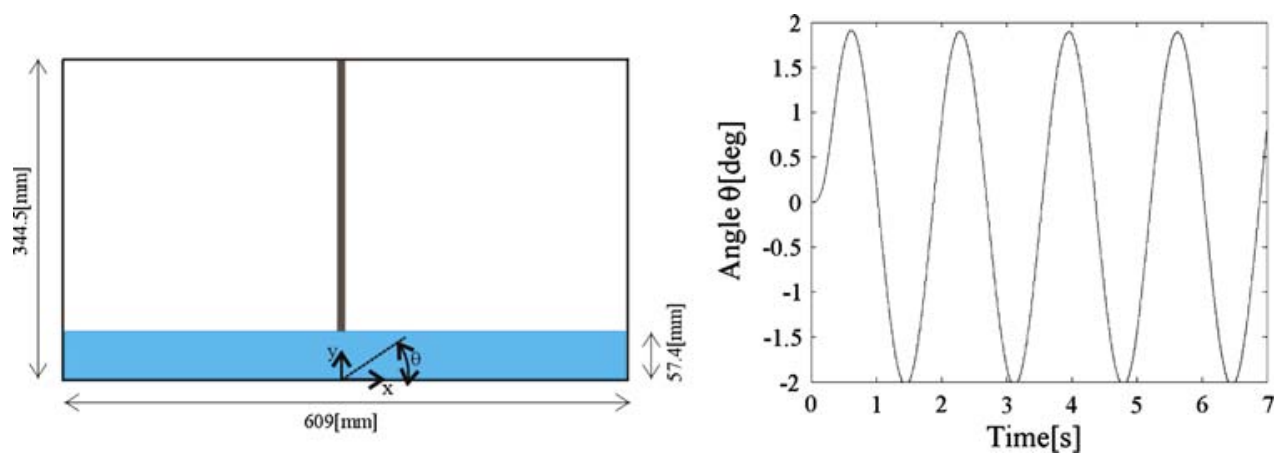
Fig. 11 Hanging elastic beam in shallow water: experimental versus numerical comparison

$t=0.76,1.64,2.4,2.68,2.96$

$3.32,3.4,3.56,3.80,3.84,4,4.16$ [s]
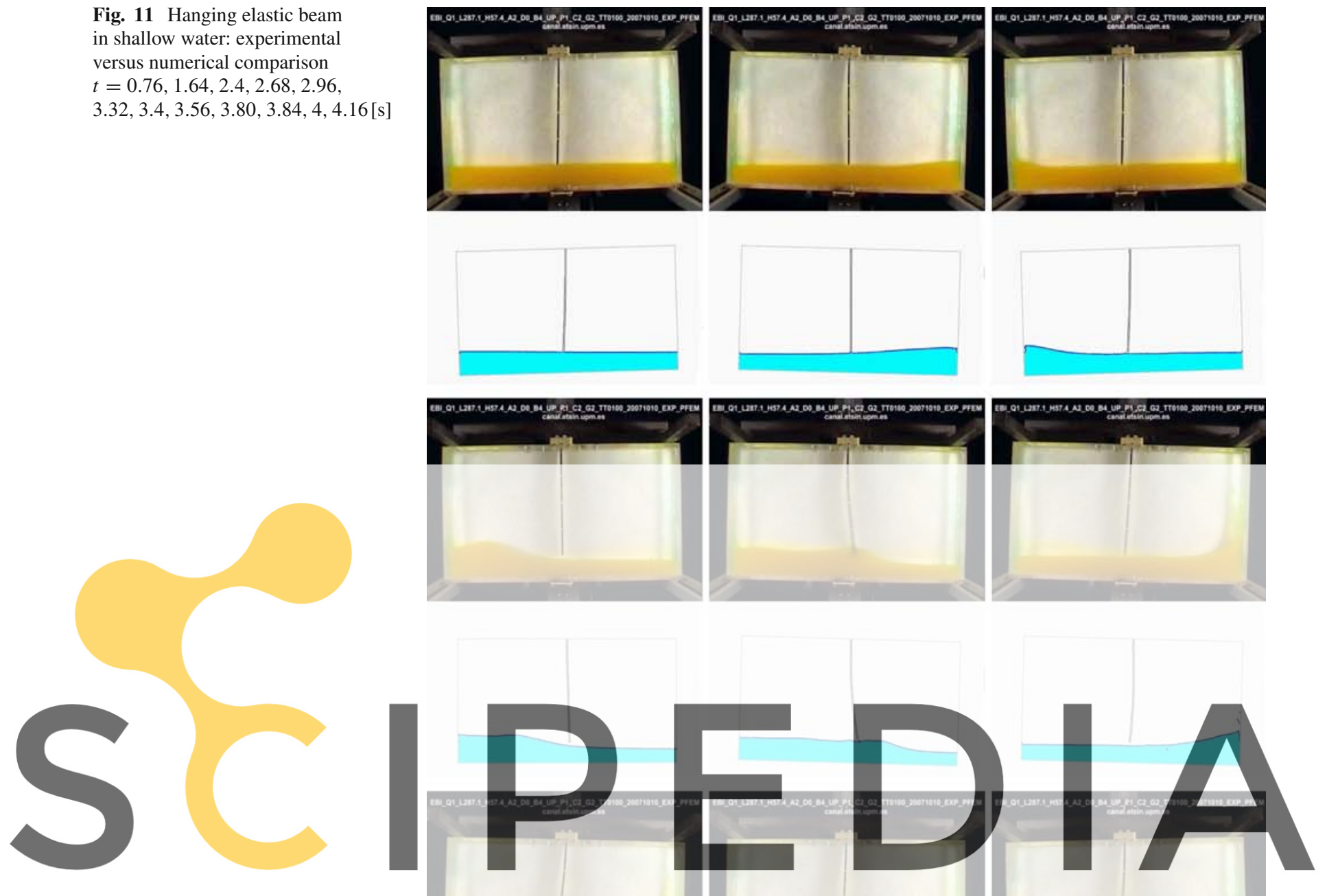

Register for free at https//www.scipedia.com to download the version without the watermark
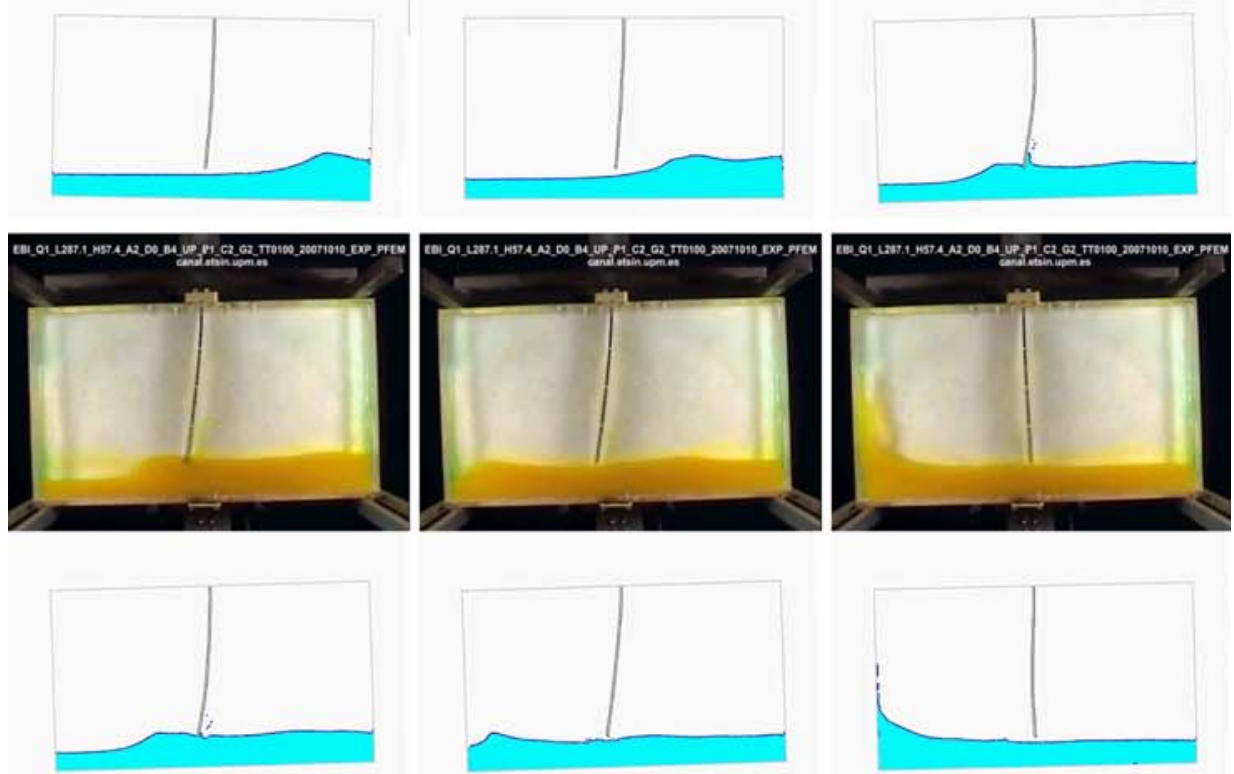
Fig. 12 Hanging elastic beam in shallow water: comparison of the end and midpoint displacement in $X$ direction
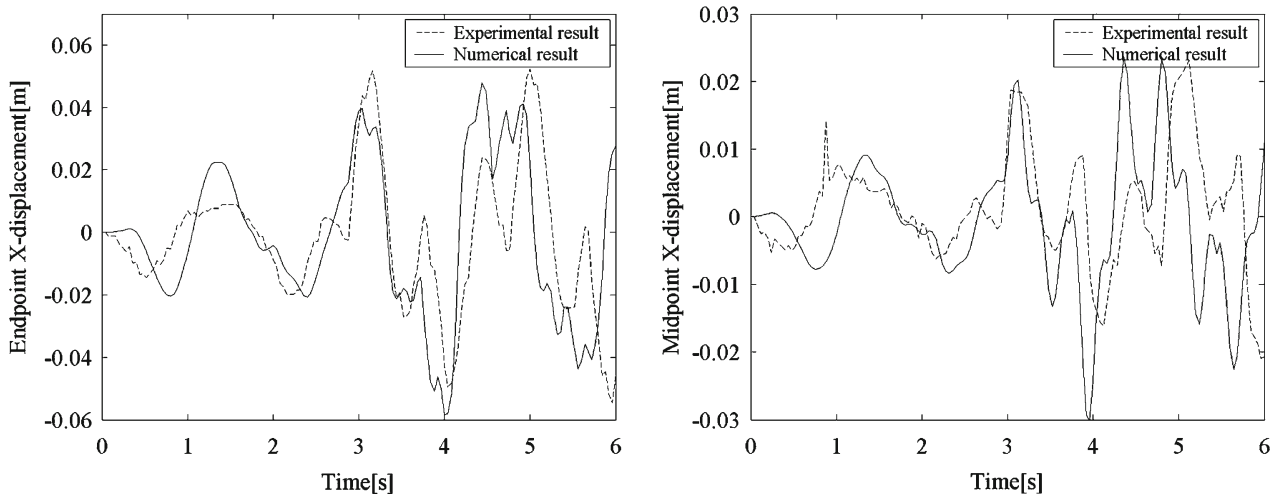

In this example the natural frequency of the free surface wave does not coincide with the imposed frequency of the beam. This produces a strong shock at the time of $2 \mathrm{~s}$. were the FSI start. High frequencies of the elastic beam are induced at this moment. Figure 12 shows the displacement of endpoint and midpoint of the beam as a function of time. The agreement between the numerical results and the experimental ones are very good, taking in account the complexity of the example.
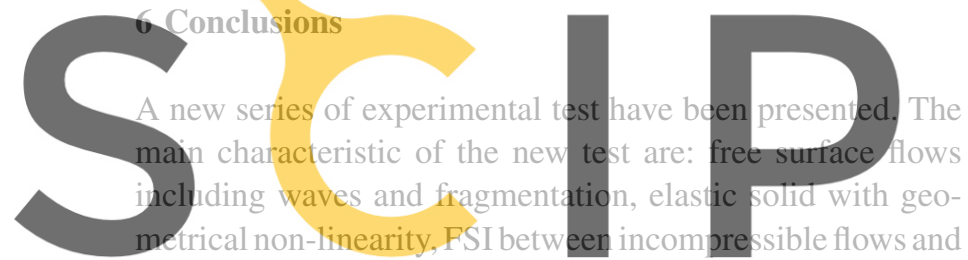
elastic beams. The experimental results have been compared Register for free at https//WWw. Scipedia.com tor to deformation of the fluid domain. Very good results have been obtained for the relevant parameters for the flow fieid and the elastic structure analyzed (such as the free surface and elastic beam position) as shown in the comparison with experimental data.

Acknowledgments This works has been supported by the $\mathrm{Al} \beta$ an Programme of the European Union for High Level Scholarships for Latin America, scholarship No.E06D100984AR.

\section{References}

1. Idelsohn SR, Oñate E, Del Pin F, Calvo N (2006) Fluid-structure interaction using the particle finite element method. Comput Method Appl Mech Eng 195:2100-2123

2. Tezduyar TE (2001) Finite element methods for flow problems with moving boundaries and interfaces. Arch Comput Methods Eng 8:83-130

3. Akin JE, Tezduyar TE, Ungor M (2007) Computation of flow problems with the mixed Interface-Tracking/Interface-Capturing Technique (MITICT). Comput Fluids 36:2-11

4. Cruchaga MA, Celentano DJ, Tezduyar TE (2007) A numerical model based on the mixed Interface-Tracking/Interface-Capturing
Technique (MITICT) for flows with fluid-solid and fluid-fluid interfaces. Int J Numer Methods Fluids 54:1021-1030

5. Idelsohn SR, Oñate E, Del Pin F (2004) The particle finite element method: a powerful tool to solve incompressible flows with freesurfaces and breaking waves. Int J Numer Methods Eng 61:964 984

6. Larese A, Rossi R, Oñate E, Idelsohn SR (2007) Validation of the particle finite element method (PFEM) for simulation of the free surface flows. Accepted in Int J Comput Method

7. Osher S, Fedkiw RP (2001) Level set methods: an overview and some recent reults. J Comput Phys 169:463-502

8. Osher S, Fedkiw RP (2006) Level set methods an dynamic implicit surfaces. Springer, Berlin

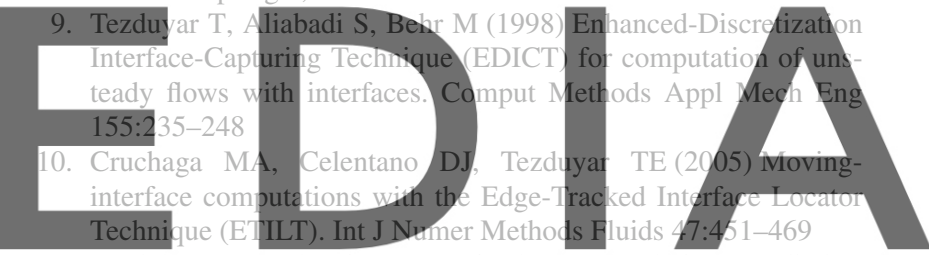

11. Roubtsova V, Kahawita R (2006) The SPH technique applied to free-surface flows. Comput Fluids 35:1359-1371

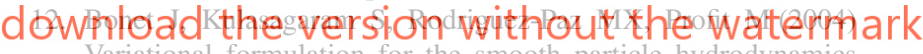
Variational formulation for the smooth particle hydrodynamics (SPH) simulation of fluid and solid problems. Comput Methods Appl Mech Eng 193:1245-1256

13. Oñate E, Idelsohn SR, Del Pin F, Aubry R (2004) The particle finite element method. An overview. Int J Comput Method 2:267-307

14. Idelsohn SR, Oñate E, Calvo N, Del Pin F (2003) The meshless finite element method. Int J Numer Methods Eng 58(6):893-912

15. Idelsohn SR, Calvo N, Oñate E (2003) Polyhedrization of an arbitrary 3D point set. Comput Methods Appl Mech Eng 192:2649_ 2667

16. Donea J, Huerta A (2003) Finite elements methods for flow problems. Wiley, New York

17. Zienkiewicz OC, Taylor R L, Nitharasu P (2005) The finite element method. Fluid dynamics, vol. III. Elsevier, Amsterdam

18. Oñate E (2000) A stabilized finite element method for incompressible viscous flows using a finite increment calculus formulation. Comput Methods Appl Mech Eng 182:355-370

19. Oñate E, Idelsohn SR (1998) A mesh free finite point method for advective diffusive transport and fluid flow problem. Comput Mech 21:283-292

20. Edelsbruner H, Mucke EP (1994) Three dimensional alpha shape. ACM Trans Graph 13:43-72

21. Souto-Iglesias A, Delorme L, Pérez-Rojas L, Abril-Pérez S (2006) Liquid moment amplitude assessment in sloshing type problems with smooth particle hydrodynamics. Ocean Eng 33:1462-1484 